\title{
CARACTERÍSTICAS CLÍNICAS E LABORATORIAIS DA COVID-19: UMA ANÁLISE NA INTERNAÇÃO HOSPITALAR
}

\section{CLINICAL AND LABORATORY CHARACTERISTICS FOR COVID-19: ANALYSIS OF HOSPITAL INTERNMENT}

\section{Letícia Silveira Goulart ${ }^{1} *$ Kassila Conceição Ferreira Santos ${ }^{2} *$ Débora Aparecida da Silva Santos $^{3} *$ Magda de Mattos $^{4}$}

\begin{abstract}
RESUMO
Objetivo: Analisar as características clínicas e laboratoriais de pacientes com COVID-19 na internação hospitalar. Métodos: Foram incluídos no estudo pacientes com diagnóstico de COVID19 internados em uma Unidade de Pronto Atendimento e em um Hospital Municipal de referência para a doença. Os dados foram coletados dos prontuários eletrônicos dos pacientes. Aplicou-se a estatística descritiva. Resultados: Foram incluídos 205 pacientes. Os sinais e sintomas clínicos mais frequentes foram dispneia $(48,29 \%)$, tosse $(30,73 \%)$ e mialgia $(24,39 \%)$. A hipertensão arterial sistêmica foi a comorbidade predominante $(73,47 \%)$. A maioria dos pacientes apresentou linfopenia $(73,12 \%)$, elevação na proteína C reativa $(97,53 \%)$, aumento de desidrogenase láctica $(94,52 \%)$ e redução na pO2 $(69,00 \%)$. Conclusões: Os dados gerados possibilitaram determinar o perfil clínico e laboratorial de pacientes com COVID-19 na internação hospitalar. Esses resultados podem contribuir para uma melhor compreensão da patogenia da doença.
\end{abstract}

Palavras-chave: COVID-19; Sinais e Sintomas; Testes Laboratoriais; Biomarcadores

\begin{abstract}
Objective: To analyze the clinical and laboratory characteristics of patients with COVID-19 in hospitalization. Methods: Patients diagnosed with COVID-19 and admitted in a Health Care Unit and in a specialized Municipal Hospital were included. Data were retrieved from the patients' electronic charts and descriptive statistics were employed. Results: 205 patients were included. The most frequent clinical symptoms comprised dyspnoea (48.29\%), coughs $(30.73 \%)$ and myalgia $(24.39 \%)$. Systemic arterial hypertension was the dominant co-morbidity (73.47\%). Most patients also had lymphopenia $(73.12 \%)$, rise in reactive protein $\mathrm{C}(97.53 \%)$, increase in lactic dehydrogenase (94.52\%) and decrease in pO2 (69.00\%). Conclusions: Data retrieved determined the clinical and laboratory profile of patients with COVID-19 at hospitalization. Results may contribute towards a better analysis of the disease's pathogeny.
\end{abstract}

Keywords: COVID-19; Signs and Symptoms; Diagnoses, Laboratory; Biomarkers

\footnotetext{
${ }^{1}$ Universidade Federal de Rondonópolis. E-mail: leticia@ ufr.edu.br - Orcid: https://orcid.org/0000-0003-1452-4908

${ }^{2}$ Secretaria Municipal de Saúde de Rondonópolis. E-mail: kassilaenf@ hotmail.com - Orcid: https://orcid.org/0000-0003-1789-7881

${ }^{3}$ Universidade Federal de Rondonópolis. E-mail: debora.santos@ufr.edu.br - Orcid: https://orcid.org/0000-0003-1862-7883

${ }^{4}$ Universidade Federal de Rondonópolis. E-mail: magda.mattos@ufr.edu.br - Orcid: https://orcid.org/0000-0001-8330-1084
} 


\section{INTRODUÇÃO}

Ao final de 2019 na cidade de Wuhan, na província de Hubei, na China, uma nova espécie de Coronavírus foi identificada após um surto de pneumonia de etiologia desconhecida, posteriormente, o agente etiológico foi denominado de Coronavírus da Síndrome Respiratória Aguda Grave 2 $\left(\right.$ SARS-CoV-2) ${ }^{1}$. Em fevereiro de 2020, a síndrome respiratória aguda grave que se alastrava pelo mundo foi nomeada pela Organização Mundial de Saúde como Doença do Coronavíurs 2019 (COVID-19) e em 11 de março do mesmo ano, a doença foi caracterizada como pandemia ${ }^{2}$.

Estima-se que a maioria dos indivíduos com COVID-19 seja assintomática ou apresente apenas sintomas leves, incluindo febre, fadiga, tosse e mialgia. Pode haver anosmia, ageusia, náusea, cefaléia, vômito, dor abdominal, diarréia, odinofagia e rinorréia. Os casos graves podem incluir dispnéia, cianose, taquipnéia, hipotensão, descompensação de doenças de base, linfopenia e necessitam de internação hospitalar ${ }^{3,4,5}$. As complicações mais comuns são Síndrome Respiratória Aguda Grave, lesão cardíaca aguda e infecção secundária ${ }^{6}$.

A semelhança dos sintomas da COVID-19 com a de outras patologias associadas ao trato respiratório superior e inferior dificulta o diagnóstico inicial da doença. Desse modo, os testes laboratoriais, realizados na fase inicial da doença, como a
Transcrição Reversa seguida de Reação em Cadeira da Polimerase (RT-PCR) que possibilita a identificação do RNA do SARS$\mathrm{CoV}-2$ e testes que detectam antígenos virais na secreção nasal são importantes para nortear a conduta clínica ${ }^{7}$.

Diferentes características clínicas e laboratoriais são observadas entre os pacientes acometidos pela COVID-19. Com isso, o monitoramento dinâmico de exames laboratoriais pode ser significativo para prever o prognóstico dos pacientes, sobretudo porque a doença é associada a um processo inflamatório grave com disfunção de órgãos ${ }^{8}$. Os parâmetros laboratoriais consideráveis para monitorar a progressão da COVID-19 incluem desidrogenase láctica, prolactina, proteína C reativa e citocinas pró inflamatórias, além de biomarcadores como linfócitos, neutrófilos, fatores de coagulação, dímero D e oximetria ${ }^{9,10}$.

Sendo assim, conhecer as alterações em exames laboratoriais e os sinais e sintomas apresentados por pacientes acometidos com a COVID-19 na internação hospitalar, pode ser uma útil ferramenta para compreender a evolução da doença, e com isso propor estratégias de cuidados e condutas adequados a esses pacientes.

\section{OBJETIVO}

O objetivo do presente estudo foi analisar as características clínicas e 
laboratoriais de pacientes com COVID-19 na internação hospitalar.

\section{MÉTODOS}

Trata-se de estudo observacional e retrospectivo, com pacientes diagnosticados com COVID-19, internados em uma Unidade de Pronto Atendimento (UPA) e em um Hospital Municipal de referência para atendimentos a COVID-19 no Município de Rondonópolis, MT, no período entre janeiro a abril de 2021.

Foram incluídos no estudo os indivíduos com confirmação laboratorial para a doença por RT-PCR ou por teste rápido de antígeno a partir de amostras de swabs nasofaríngeos no período de estudo. Foram excluídos aqueles pacientes cujos dados nos prontuários estavam incompletos.

Foram coletados os dados clínicos e resultados dos exames laboratoriais apresentados pelos pacientes no momento de sua internação nas instituições de saúde. As informações foram obtidas do prontuário eletrônico do paciente e transcritas para um formulário estruturado para coleta de dados.

As variáveis analisadas foram classificadas em blocos:

a) sociodemográficos (idade e sexo)

b) clínicos (comorbidades, tempo de sintomas e sintomas de COVID);

c) parâmetros hematológicos (leucócitos totais, linfócitos, monócitos, eritrócitos, hemoglobina, hematócrito e plaquetas) e d) parâmetros bioquímicos (Proteína C reativa, sódio, potássio, pressão parcial de gás carbônico $\left(\mathrm{pCO}_{2}\right)$, pressão parcial de Oxigênio ( $\left.\mathrm{pO}_{2}\right), \mathrm{pH}$ e bicarbonato).

Os dados foram tabulados no programa Microsfot Excel 2013 e foram analisados pelo programa JASP. Realizou-se a estatística descritiva. As variáveis contínuas foram expressas em média com desvio padrão, mediana, valores mínimo e máximo. As variáveis categóricas foram expressas em frequência absoluta e relativa.

Apesar de se tratar de dados secundários, esta pesquisa foi aprovada pelo Comitê de Ética e Pesquisa do Hospital Universitário Júlio Muller, Universidade Federal de Mato Grosso, CAEE 40583220.7.0000.5165, Número do Parecer: 4.418.798.

\section{RESULTADOS}

Foram incluídos no presente estudo 205 pacientes, $111(54,15 \%)$ do sexo masculino, idade média 57,13 anos (Desvio padrão: 17,41, Mínimo=13 e Máximo=94). Os sinais e sintomas clínicos mais frequentes na população estudada foram dispneia $(48,29 \%)$, tosse $(30,73 \%)$, mialgia $(24,39 \%)$ e febre $(20,00 \%)$. O período médio de surgimento dos sinais e sintomas anterior a internação hospitalar foi 8,3 dias.

A prevalência de comorbidades nos pacientes hospitalizados por COVID-19 foi 47,80\%. As comorbidades mais frequentes 
foram hipertensão arterial sistêmica $(73,47 \%)$, diabetes mellitus $(31,63 \%)$ e obesidade $(13,26 \%) . \quad$ A tabela 1 descreve as

Tabela1 - Características sociodemográficas e clínicas de indivíduos com COVID-19. Rondonópolis, MT, 2021.

\begin{tabular}{|c|c|}
\hline Variável & $\mathbf{N}(\%)$ \\
\hline \multicolumn{2}{|l|}{ Gênero } \\
\hline Feminino & $94(45,85)$ \\
\hline Masculino & $111(54,15)$ \\
\hline \multicolumn{2}{|l|}{ Idade (anos) } \\
\hline Mediana (Min - Máx) & $57(13-94)$ \\
\hline 13 a 39 & $29(14,14)$ \\
\hline 40 a 59 & $91(44,39)$ \\
\hline 60 ou mais & $85(41,46)$ \\
\hline \multicolumn{2}{|l|}{ Comorbidades } \\
\hline Sim & $98(47,81)$ \\
\hline Não & $107(52,19)$ \\
\hline Hipertensão arterial sistêmica & $72(73,47)$ \\
\hline Diabetes mellitus & $31(31,63)$ \\
\hline Obesidade & $13(13,26)$ \\
\hline Cardiopatia & $7(7,14)$ \\
\hline Câncer & $3(3,06)$ \\
\hline Alzheimer & $1(1,02)$ \\
\hline Hanseníase & $1(1,02)$ \\
\hline Epilepsia & $1(1,02)$ \\
\hline Hanseníase & $1(1,02)$ \\
\hline Transtorno psiquiátrico & $1(1,02)$ \\
\hline \multicolumn{2}{|c|}{ Duração dos sintomas antes da admissão em dias } \\
\hline Média & 8,3 \\
\hline Até 7 & $86(42,00)$ \\
\hline 8 a 14 & $108(53,00)$ \\
\hline 15 ou mais & $11(5,36)$ \\
\hline
\end{tabular}

\section{Sinais e sintomas clínicos na admissão}


Dispneia

Tosse

Mialgia

Febre

Astenia

Cefaleia

Anosmia

Ageusia

Náuseas e vômito
$99(48,29)$

$63(30,73)$

$50(24,39)$

$41(20)$

$5(17,07)$

$23(11,21)$

$17(8,29)$

$13(6,34)$

$17(8,29)$

Fonte: Os autores

A análise dos dados hematológicos revelou que a maioria dos casos apresentou valores dentro da normalidade para a contagem de leucócitos totais $(68,29 \%)$, monócitos $(88,44 \%)$, eritrócitos $(81,00 \%)$ e plaquetas $(88,67 \%)$, assim como para os valores do hematócrito $(80,00 \%)$ e hemoglobina $(84,39 \%)$. Uma linfopenia foi observada na maioria dos pacientes $(73,13 \%)$, com uma mediana de 496,00 células por $\mathrm{mm}^{3}$ (Tabela 2).

Tabela 2 - Parâmetros hematológicos de indivíduos com COVID-19. Rondonópolis, MT, 2021.

\begin{tabular}{|c|c|}
\hline Variável & $\mathbf{N}(\%)$ \\
\hline \multicolumn{2}{|c|}{ Contagem de leucócitos $\left(4.000\right.$ - $\left.11.000 / \mathrm{mm}^{3}\right)$} \\
\hline Mediana por $\mathrm{mm}^{3}$ & 7.520 \\
\hline Normal & $120(68,29)$ \\
\hline Elevada & $52(25,36)$ \\
\hline Reduzida & $13(6,34)$ \\
\hline \multicolumn{2}{|c|}{ Contagem de linfócitos $\left(1.000\right.$ - $\left.4.950 / \mathrm{mm}^{3}\right)$} \\
\hline Mediana por $\mathrm{mm}^{3}$ & 496,00 \\
\hline Normal & $54(27,00)$ \\
\hline Elevada & $0(0,00)$ \\
\hline Reduzida & $147(73,13)$ \\
\hline \multicolumn{2}{|c|}{ Contagem de monócitos (80 - 1.100 /mm³) } \\
\hline Mediana por $\mathrm{mm}^{3}$ & 377,00 \\
\hline Normal & $176(88,44)$ \\
\hline Elevada & $17(8,54)$ \\
\hline
\end{tabular}


Reduzida

$6(3,01)$

Contagem de eritrócitos (4,00 - 5,40 milhões $\left./ \mathrm{mm}^{3}\right)$

Mediana milhões por $\mathrm{mm}^{3}$

Normal

$166(81,00)$

Elevada

$1(0,48)$

Reduzida

$38(18,53)$

Hematócrito (37-45\%)

Mediana

40,60

Normal

$164(80,00)$

Elevado

$2(0,97)$

Reduzido

$39(19,02)$

Dosagem de Hemoglobina (11,7 - 16,0 g/dL)

Mediana $\mathrm{g} / \mathrm{dL}$

Normal

$173(84,39)$

Elevada

$1(0,48)$

Reduzida

Contagem de plaquetas $\left(150.000\right.$ a $\left.450.000 / \mathrm{mm}^{3}\right)$

Mediana por $\mathrm{mm}^{3}$

Normal

$180(88,67)$

Elevada

$2(0,98)$

Reduzida

$21(10,34)$

Fonte: Os autores

Os pacientes estudados apresentaram na admissão hospitalar níveis aumentados de PCR $(97,53 \%)$ e desidrogenase láctica $(94,52 \%)$, bem como, a redução da pO2 (69,00\%). Os valores de sódio, potássio, bicarbonato, pH e pCO2 apresentaram-se normais para a maioria dos casos (Tabela $3)$.

Tabela 3 - Parâmetros bioquímicos de indivíduos com COVID-19. Rondonópolis, MT, 2021.

\begin{tabular}{ll}
\hline Variável & N (\%) \\
\hline Proteína C Reativa (Menor que 6 mg/L) & \\
Mediana em mg/L & 102,90 \\
Normal & $5(2,46)$ \\
Elevada & $198(97,53)$
\end{tabular}


Reduzida

$0(0,00)$

\section{Sódio (136 a $145 \mathrm{mmol} / \mathrm{L}$ )}

Mediana em mmol/L

137,00

Normal

$179(91,00)$

Elevado

$4(2,03)$

Reduzido

$14(7,10)$

Potássio (3,6 a 5,5 mmol/L)

Mediana em mmol/L

4,30

Normal

$176(90,25)$

Elevado

Reduzido

$12(6,15)$

Desidrogenase láctica (135 a 225 U/L)

Mediana em U/L

409,00

Normal

$4(5,479)$

Elevado

$67(94,52)$

Reduzido

$0(0,00)$

pCO$_{2}$ (35 a 45 mmHg)

Mediana em mmHg

35,70

Normal

$91(52,29)$

Elevado

$16(9,19)$

Reduzido

$67(35,50)$

$\mathrm{pO}_{2}$ (83 a $\left.108 \mathrm{mmHg}\right)$

Mediana em $\mathrm{mmHg}$

65,15

Normal

$39(22,41)$

Elevado

$15(8,62)$

Reduzido

$120(69,00)$

Bicarbonato (21 - $28 \mathrm{Mol} / \mathrm{L}$ )

Mediana em Mol/L

23,05

Normal

$121(69,54)$

Elevado

$8(4,59)$

Reduzido

$45(26,00)$

pH $(7,32$ a 7,43)

Mediana

7,42

Normal

$137(79,65)$ 
Elevado

Reduzido

$12(7,00)$

Fonte: Os autores

\section{DISCUSSÃO}

Os pacientes com COVID-19 incluídos na presente pesquisa apresentaram idade média de 57,13 anos, com predomínio do gênero masculino $(54,15 \%)$. A análise do perfil demográfico de pacientes com diagnóstico de COVID-19 em um hospital público de referência na cidade de Fortaleza, Ceará, Brasil, revelou que maioria dos pacientes era do gênero masculino, pertencentes à faixa etária de 36 a 60 anos ${ }^{11}$. Um estudo retrospectivo com 1335 pacientes hospitalizados por COVID-19 em Londres, Inglaterra, indicou que a média de idade foi de 70 anos e $56 \%$ eram homens ${ }^{12}$. Uma pesquisa realizada na Escócia verificou que a média de idade dos pacientes hospitalizados por COVID-19 foi de 76 anos e $52,70 \%$ correspondiam ao gênero masculino ${ }^{13}$.

Um estudo que determinou os fatores associados ao risco ou à proteção para à COVID-19 no sul do Brasil demonstrou que as prevalências de internação por COVID-19 são menores para o sexo feminino. Os autores sugerem que diferenças biológicas entre homens e mulheres devem refletir em respostas imunológicas distintas, impactando no curso da doença ${ }^{14}$. Deve-se também considerar a suscetibilidade genética individual e as influências ambientais na infecção do vírus, o que pode resultar em diferentes fenótipos clínicos entre populações e países ${ }^{15}$.

De acordo com o Ministério da Saúde, febre, tosse, dispneia, mialgia e fadiga são considerados os sinais e sintomas mais comuns na COVID-19 ${ }^{16}$. Na população estudada, observou-se um predomínio de dispneia, tosse e febre. Liu et al., descreveram que febre, tosse e fadiga foram os sintomas mais prevalentes nos casos de internação por COVID-19 ${ }^{17}$. No estudo de Teich e colaboradores as manifestações clínicas mais comuns em pacientes hospitalizados por COVID-19 foram cefaleia, tosse e congestão nasal $^{4}$. As variações nas manifestações clínicas são decorrentes, entre outras propriedades, de diferenças na faixa etária, morbidades, condições sociais, culturais e cuidados de saúde. Identificar as principais características clínicas dos pacientes infectados por SARS-CoV-2 poderá contribuir no manejo da doença ${ }^{18}$.

As comorbidades mais frequentes foram hipertensão arterial sistêmica e diabetes mellitus, corroborando com estudos prévios $^{4,12,13}$. A presença de comorbidades está associada ao desenvolvimento de COVID-19 grave e ao maior risco de óbito ${ }^{9,14,19}$. A infecção por SARS-CoV-2 é 
desencadeada quando a proteína $\mathrm{S}$ do vírus liga-se à enzima conversora de angiotensina 2 (ECA2), resultando em acúmulo de angiotensina 2 e redução de angiotensina 1-7. $\mathrm{O}$ papel da angiotensina $2 \mathrm{em}$ pacientes hipertensos COVID-19 parece ser crucial pois promove vasoconstrição, retenção de sódio, estresse oxidativo, inflamação e fibrose, comprometendo a regulação da pressão arterial $^{20}$. A expressão de ECA2 está aumentada em pacientes com diabetes mellitus tipo 2. Esta regulação positiva está associada com inflamação crônica, ativação de células endoteliais e resistência à insulina o que que agrava a resposta inflamatória e leva à disfunção da barreira alvéolo-capilar ${ }^{9}$.

A COVID-19 é uma infecção sistêmica com impacto significativo no sistema hematopoiético e na hemostasia. Dentre as alterações hematológicas, a linfopenia pode ser considerada um importante achado laboratorial, com relevância para o prognóstico da doença ${ }^{21,22}$. A maioria (73\%) dos pacientes analisados apresentaram linfopenia, como descrito em estudos prévios ${ }^{4,23,24,25}$. Indivíduos com COVID-19 grave ou crítica apresentam uma contagem mais baixa de linfócitos em relação a pacientes com doença não grave ${ }^{24}$. O estudo caso-controle de Pan et al., identificou que linfopenia foi um fator independente, associado à mortalidade em indivíduos com COVID-19 grave $^{8}$. A redução na contagem de linfócitos pode ser resultado da ligação viral às células com subsequente lise, exsudação de linfócitos circulantes para tecidos pulmonares e atrofia de órgãos linfoides o que prejudica a renovação celular ${ }^{9,21}$.

A análise dos exames laboratoriais revelou elevação nos níveis de PCR em 97,04\% dos casos. Dentre os marcadores relacionados com resposta à reação inflamatória de fase aguda, a PCR é a mais sensível, porém com baixa especificidade. A proteína ativa o sistema complemento pela via clássica, inicia a opsonização, promove a quimiotaxia e por fim, estimula os processos de fagocitose e lise dos antígenos ${ }^{26}$. Uma frequência de $88 \%$ de pacientes iranianos internados em um hospital de referência para COVID-19 apresentou elevação nos níveis de $\mathrm{PCR}^{25}$. Um estudo conduzido em um hospital de São Paulo - SP, observou que 93\% dos pacientes internados por COVID-19 apresentavam aumento de $\mathrm{PCR}^{4}$. Em pacientes com COVID-19 internados em um hospital de Wuhan, China, a elevação dos níveis de PCR foi associada com maior severidade da doença, os valores médios mais elevados foram observados na fase de progressão da infecção ${ }^{23}$.

Outra alteração laboratorial observada foi a redução nos níveis de pO2, esse achado também foi descrito em outras pesquisas ${ }^{8,23}$. Um estudo de coorte multicêntrico englobando hospitais da Europa e Estados Unidos verificou que dentre outros fatores, a redução nos níveis de saturação de $\mathrm{O} 2 \leq 93 \%$ 
foi associada à maior mortalidade por COVID-1927. A principal manifestação clínica apresentada pelos pacientes estudados foi a dispneia, o que pode ser justificado pela frequente observação de redução na $\mathrm{pO} 2 . \mathrm{O}$ acompanhamento da hipoxemia nesses pacientes é fundamental para a tomada de decisões, tanto quanto para a orientação do tratamento, quanto para avaliar o prognóstico da infecção ${ }^{28}$.

O presente estudo apresenta algumas limitações, como informações incompletas em alguns prontuários, sobretudo, os dados de desidrogenase láctica, que não estavam disponíveis para a maioria dos pacientes. Vale ressaltar que essa limitação é frequente em estudos retrospectivos e que utilizam registros de prontuários. Estudos futuros englobando outros exames laboratoriais devem ser realizados a fim de contribuir com a melhor compreensão da COVID-19.

\section{CONCLUSÕES}

Os dados apresentados permitiram definir o perfil clínico e laboratorial de pacientes internados por COVID-19 na região sul de Mato Grosso. A manifestação clínica mais frequente foi dispneia e hipertensão arterial sistêmica foi a comorbidade mais prevalente. Linfopenia, elevação de PCR, desidrogenase láctica e redução de pO2 foram as alterações laboratoriais observadas. Esses resultados podem contribuir para uma melhor compreensão da epidemiologia da COVID19.

\section{REFERÊNCIAS}

1. Hamid S, Mir MY, Rohela GK. Novel coronavirus disease (COVID-19): a pandemic (epidemiology, pathogenesis and potential therapeutics). New Microb. New Infect. 2020; 35:100679.

2. Organização Mundial de Saúde. Folha informativa - COVID-19 (doença causada pelo novo coronavírus). Disponível em: https://www.paho.org/bra/index.php?option=c om content\&view=article\&id=6101: covid 19 \&Itemid $=875$

3. Xu X, Wu XX, Jiang XG. et al. Clinical findings in a group of patients infected with the 2019 novel coronavirus (SARS-Cov-2) outside of Wuhan, China: retrospective case series. BMJ 2020; 368:m606.

4. Teich VD, Klajner S, Almeida FAS, et al. Características epidemiológicas e clínicas dos pacientes com COVID-19 no Brasil. Einstein. 2020; 18: 1-7.

5. Cespedes MS, Souza JCRP. Sars-CoV-2: A clinical update - II. Rev. Assoc. Med. Bras. 2020; 66 (4): 547-557.

6. Lippi G, Sanchis-gomar F, Brandon M, Henry BM. COVID-19: unravelling the clinical progression of nature's virtually perfect biological weapon. Ann Transl Med, v. 8, n. 11, p. 1-6, 2020.

7. Iser BPM, Silva I, Raymundo VT, Poleto MB, Schuelter-Trevisol F, Bobinski F. Definição de caso suspeito da COVID-19: uma revisão narrativa dos sinais e sintomas mais frequentes entre os casos confirmados. Epidemiol. Serv. Saúde. 2020; 29 (3): e2020233.

8. Pan F, Yang L, Li Y, et al. Factors associated with death outcome in patients with severe coronavirus disease-19 (COVID19): a case-control study. Int. J. Med. Sciences, 2020; 17 (9): 1281-92. 
9. Gao Y, Ding M, Dong X, et al. Risk factors for severe and critically ill COVID-19 patients: A review. Allergy, 2021; 76: 428455.

10. Tjendra Y, Al Mana AF, Espejo AP. Predicting Disease Severity and Outcome in COVID-19 Patients. Arch Pathol Lab Med. 2020; 144; 1465-74.

11. Rebouças ERN, Costa RF, Miranda LR, Campos NG. Perfil demográfico e clínico de pacientes com diagnóstico de COVID-19 em um hospital público de referência na cidade de Fortaleza-Ceará. J. Health Biol Sci. 2020; 8 (1): $1-5$.

12. Zakeri R, Picklesc A, Carrc E. et al. Biological responses to COVID-19: Insights from physiological and blood biomarker profiles. Cur. Res. Trans. Med. 2021; 69: 103276.

13. Hetherington L, Johnston B, Kotronoulas G, Finlay F, Keeley P, McKeown A. COVID-19 and Hospital Palliative Care - A service evaluation exploring the symptoms and outcomes of 186 patients and the impact of the pandemic on specialist Hospital Palliative Care. Palliat. Med. 2020; 34 (9): 1256-62.

14. Klokner SGM, Luz RA, Araujo PHM, et al. Perfil epidemiológico e preditores de fatores de risco para a COVID-19 na região sul do Brasil. Res. Soc. Develop. 2021; 10 (3): e17710313197.

15. Gemmati D, Bramanti B, Serino ML, P Secchiero P, Zauli G, Tisato V. COVID-19 and Individual Genetic Susceptibility/Receptivity: Role of ACE1/ACE2 Genes, Immunity, Inflammation and Coagulation. Might the Double XChromosome in Females Be Protective against SARS-CoV-2 Compared to the Single X-Chromosome in Males? Int. J. Mol. Sci. 2020; 21: 3474.

16. Brasil. Ministério da Saúde. Protocolo de Manejo Clínico para o Novo Coronavírus (2019-nCoV). Brasília, DF, 2020.

17. Liu Y, Mao B, Liang S, et al. Association between age and clinical characteristics and outcomes of COVID-19. Eur Respir J. 2020; 55: 2001112

18. Santos PSA, Mateus SRM, Silva MFO, Figueiredo PTS, Campolino RG. Perfil epidemiológico da mortalidade de pacientes internados por Covid-19 na unidade de terapia intensiva de um hospital universitário. Braz. J. Develop. 2021; (7): 5: 45981-92.

19. Galvão, MHR , Roncalli AG. Fatores associados a maior risco de ocorrência de óbito por COVID-19: análise de sobrevivência com base em casos confirmados. Rev Bras Epidemiol. 2020; 23: E200106.

20. Morbio AP, Fonseca Filho PR. Comorbidades e os achados radiográficos em pacientes com COVID-19. J. Health NPEPS. 2021 jan-jul; 6(1):e5510.

21. Fleury MK. A COVID-19 e o laboratório de hematologia: uma revisão da literatura recente. RBAC. 2020; 52 (2): 131-7

22. Careli GZ. Alterações laboratoriais em pacientes com COVID-19. Res. Soc. Develop. 2020; 9 (12): e30191211115.

23. Zhang B, Zhang J, Chen H, et al. Novel coronavirus disease 2019 (COVID-19): relationship between chest CT scores and laboratory parameters. Europ. J. Nuc. Med. Mol. Imag. 2020; 47: 2083-89.

24. Wang $F$, Nie J, Wang $\mathrm{H}$, et al. Characteristics of Peripheral Lymphocyte Subset Alteration in COVID-19 Pneumonia. J Infect Dis. 2020. 11; 221 (11): 1762-69.

25. Rokni M, Ahmadikia K, Asghari S, Mashaei S, Hassanali F. Comparison of clinical, para-clinical and laboratory findings in survived and deceased patients with COVID-19: diagnostic role of inflammatory indications in determining the severity of illness. BMC Infect Dis. 2020; 20(1):869.

26. Junior RB, Lourenço PM. Alterações laboratoriais e a COVID-19. RBAC. 2020; 52(2):198-200.

27. Bertsimas D, Lukin G, Mingardi L, et al. COVID-19 mortality risk assessment: An international multi-center study. PLoS ONE. 15 (12): e0243262. 
28. Dhont S, Derom E, Braeckel EV, Depuydt P, Lambrecht BN. The pathophysiology of 'happy' hypoxemia in COVID-19. Resp. Res. 2020. 21 (198): 1-9.

Submissão: 2021-08-23

Aprovado: 2021-10-26 